\title{
Vector Vortex Beam Generation with a Single Plasmonic Metasurface
}

Fuyong Yue ${ }^{1}$, Dandan Wen ${ }^{1}$, Jingtao Xin ${ }^{2}$, Brian D. Gerardot ${ }^{1}$, Jensen $\mathrm{Li}^{3}$, Xianzhong Chen ${ }^{\text {* }^{*}}$

1. SUPA, Institute of Photonics and Quantum Sciences, School of Engineering and Physical

Sciences,

Heriot-Watt University, Edinburgh, EH14 4AS, UK

2. Beijing Engineering Research Centre of Optoelectronic Information and Instruments, Beijing Information Science and Technology University, Beijing 100192, China

3. School of Physics and Astronomy, University of Birmingham, B15 2TT 
Supplementary Section 1. Rod distribution of metasurfaces with different $\alpha_{0}$ and polarization distribution of resultant beam reflected from metasurface produced by LCP and RCP incident light.

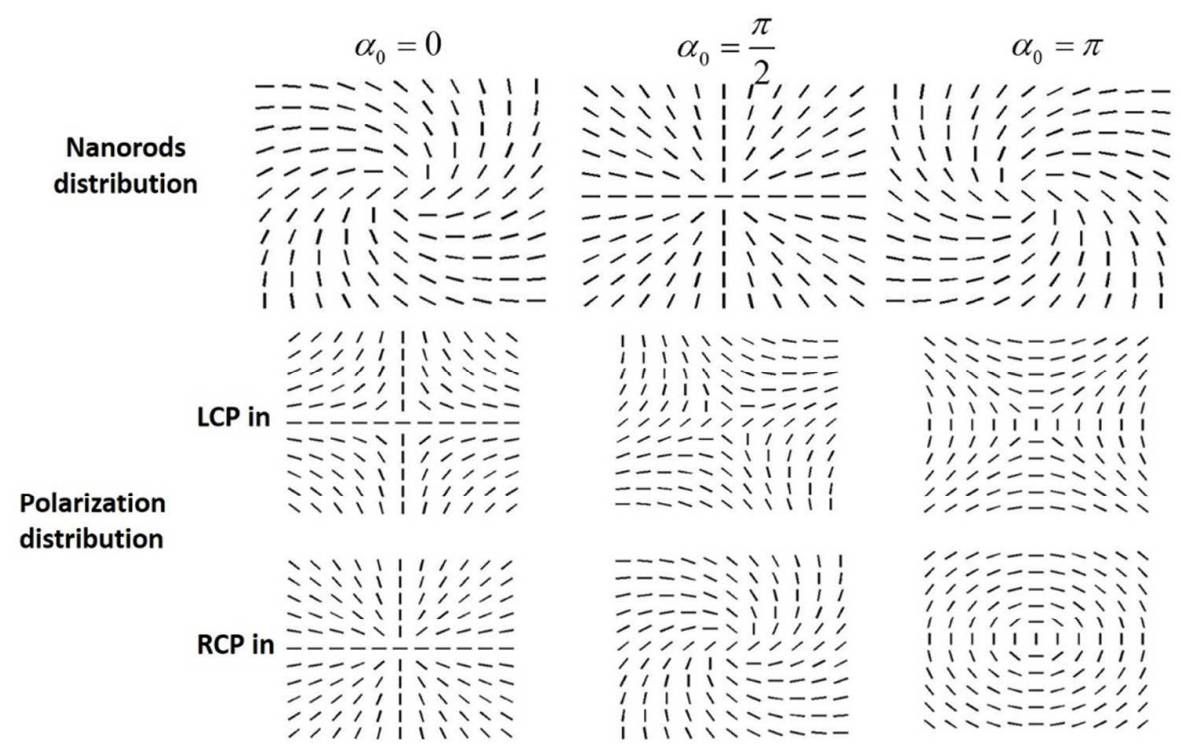

Figure S1. Nanorods distribution of metasurfaces with different $\alpha_{0}$ are shown in the first column. The polarization distributions of emerging beam for LCP and RCP incident light are illustrated in the second and third column, respectively.

Supplementary Section 2. Polarization and phase evolution on a single-pixel cell structure.

a

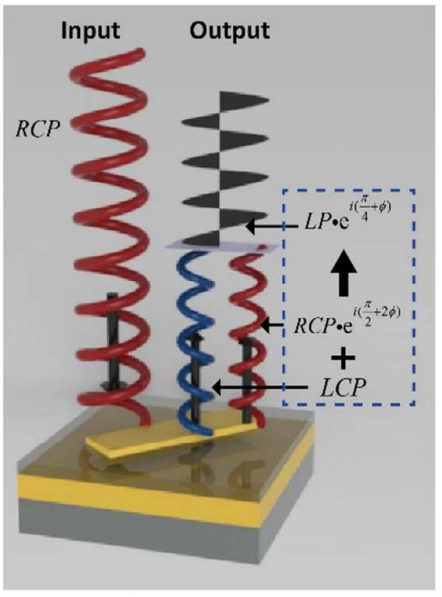

b

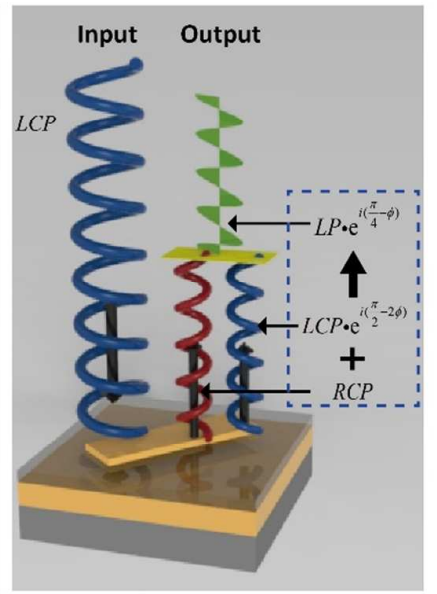

Figure S2. Illustration of polarization and phase evolution of emerging light on a single-pixel cell structure. For the circularly polarized incident light, the emerging light is the superposition of two orthogonal circularly polarized beam which are the converted wave (same handedness with that of 
incident beam) and residual wave (opposite handedness with that of incident beam), respectively. When the converted part and residual part have equal components, the output beam gives rise to linearly polarized light. Spiral curved line in red color stands for right-handed circularly polarized light (RCP), and that in blue color stands for left-handed circularly polarized light (LCP). The polarization states of the incident light are (a) RCP and (b) LCP, respectively.

A single-pixel cell structure is functionally similar with a reflective-type half-wave plate with fast axis parallel to the major axis of nanorod ${ }^{1}$ (Fig. S1). The Jones matrix of reflective-type half-wave plate is given by ${ }^{2}$

$$
J_{(\lambda / 2, \phi)}=i\left[\begin{array}{cc}
\cos 2 \phi & \sin 2 \phi \\
\sin 2 \phi & -\cos 2 \phi
\end{array}\right]
$$

where $\phi$ is the angle between the fast axis of half-wave plate and the horizontal axis. In general case, the factor $i$ is usually suppressed since we only consider the converted part. While in this case, it can't be ignored since the phase difference with regard to incident wave is the key point. When a pure circularly polarized light impinges normally upon the localized half-wave plate, the reflection beam is in general a superposition of two components with orthogonal circular polarization states, the converted part with an abrupt phase change, and the residual part without phase change. First we consider the RCP incident light. Its normalized Jones vector is

$$
E^{R C P}=\frac{1}{\sqrt{2}}\left[\begin{array}{l}
1 \\
i
\end{array}\right]
$$

The Jones vector of converted light could be derived by multiplying equations (S1) and (S2). Assuming that part of incident light is converted and we find that

$$
E_{C o n}^{R C P}=J_{(\lambda / 2, \phi)} \frac{A}{A+B} E^{R C P}=\frac{1}{\sqrt{2}} \frac{A}{A+B} e^{i\left(2 \phi+\frac{\pi}{2}\right)}\left[\begin{array}{c}
1 \\
-i
\end{array}\right]
$$

where A and B represent the amplitudes of converted and residual light, respectively. From equation (S3), we can see that the incident RCP light is converted to LCP light with an additional phase $\left(\frac{\pi}{2}+2 \phi\right)$ (Fig. S1 a). The term $2 \phi$ is known as Pancharatnam-Berry phase. The term $\frac{\pi}{2}$ is an overal phase delay incroduced by the half-wave plate. It should be noted that the handedness of polarized light is reversed when it is reflected by an ideal mirror 
because of the opposite direction of propagation. Thus the converted wave maintains the handedness of the incident light as RCP but with additional phase $\left(\frac{\pi}{2}+2 \phi\right)$. The Jones vector is

$$
E_{\text {Con }}^{R C P}=\frac{1}{\sqrt{2}} \frac{A}{A+B} e^{i\left(2 \phi+\frac{\pi}{2}\right)}\left[\begin{array}{l}
1 \\
i
\end{array}\right]
$$

The residual wave only experiences handedness flip and the Jones vector can be given by

$$
E_{\text {Res }}^{R C P}=\frac{1}{\sqrt{2}} \frac{B}{A+B}\left[\begin{array}{c}
1 \\
-i
\end{array}\right]
$$

The emerging beam is the superposition of converted ( $\left.E_{C o n}^{R C P}\right)$ and residual wave $\left(E_{R e s}^{R C P}\right)$.If the converted and residual component have equal intensity $(A=B)$, the resultant beam gives rise to linearly polarized light and also acquires a phase change. It can be expressed as follows (Fig. S1

a)

$$
E_{\text {out }}^{R C P}=\frac{1}{2}\left(E_{\text {Con }}^{L C P}+E_{\text {Non-con }}^{L C P}\right)=\sqrt{2} e^{i\left(\frac{\pi}{4}+\phi\right)}\left[\begin{array}{c}
\cos \left(\frac{\pi}{4}+\phi\right) \\
-\sin \left(\frac{\pi}{4}+\phi\right)
\end{array}\right]
$$

A similar derivation takes place when the polarization state of incident light is LCP (Fig. S1 b). The Jones vector of emerging beam is

$$
E_{\text {out }}^{L C P}=\sqrt{2} e^{i\left(\frac{\pi}{4}-\phi\right)}\left[\begin{array}{c}
\cos \left(\frac{\pi}{4}-\phi\right) \\
\sin \left(\frac{\pi}{4}-\phi\right)
\end{array}\right]
$$

The superscripts in equations (S2-S7) represent the polarization state of incident light. From equations (S6) and (S7), we can see that the resultant beam gives rise to linearly polarized light and acquires a phase change for both LCP and RCP incident light. More importantly, both the polarization angle and phase change are dependent on the orientation angle of nanorods and the handedness of incident light. In addition, an overall $\pi / 4$ shift is introduced by the half-wave plate ${ }^{3}$. 
Supplementary Section 3. Intensity distributions of vector vortex beams with different topological charges.

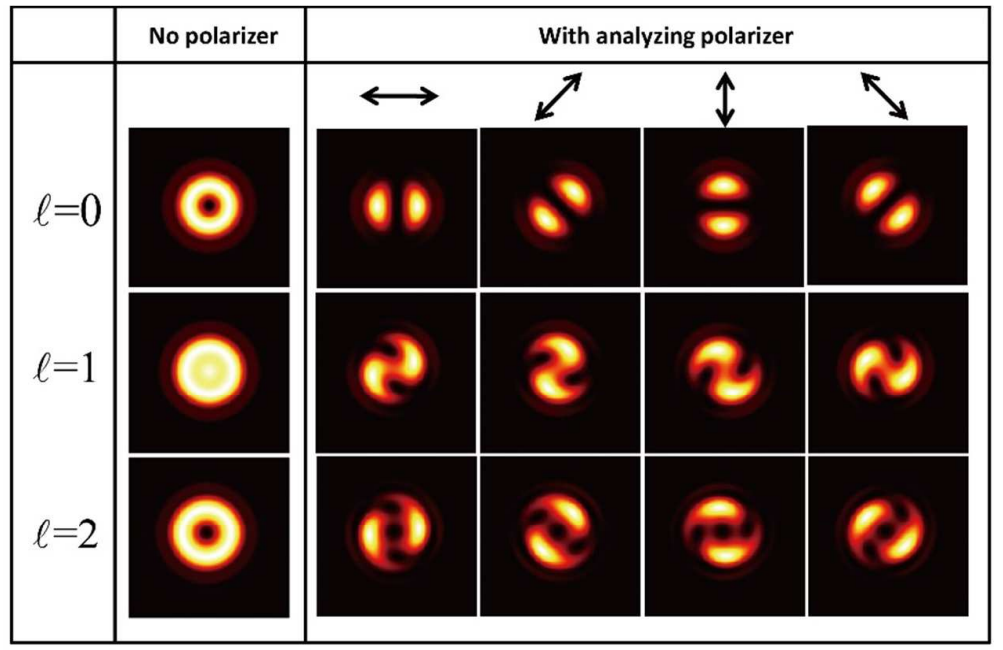

Figure S3. Simulated intensity distributions of vector beams with different topological charges $\ell=0$, $\ell=1$ and $\ell=2$. The Black double-headed arrows represent the transmission axis of linear analyzing polarizer.

\section{Supplementary Section 4. Phase gradient metasurface}

The phase gradient metasurface is based on transmission type. A single-layer of nanorods have the identical geometric parameters, which are $80 \mathrm{~nm}$ in width, $200 \mathrm{~nm}$ in length and 30 $\mathrm{nm}$ in thickness. The center-to-center distance between the two neighboring nanorods is 300 $\mathrm{nm}$. The angular orientation of each nanorod varies along the $\mathrm{x}$-direction with an increment of $\pi / 6$ in clockwise rotation, but remains invariant in the $\mathrm{y}$-direction, hence each period in $\mathrm{x}$ direction contains six nanorods whose orientations change from 0 to $\pi$, with the phase shift ranging from 0 to $2 \pi$. The SEM images of PGM is shown in Fig. S4. The calculated deflection angle based on the generalized Snell's law ${ }^{4}$ is 22.7 degrees.

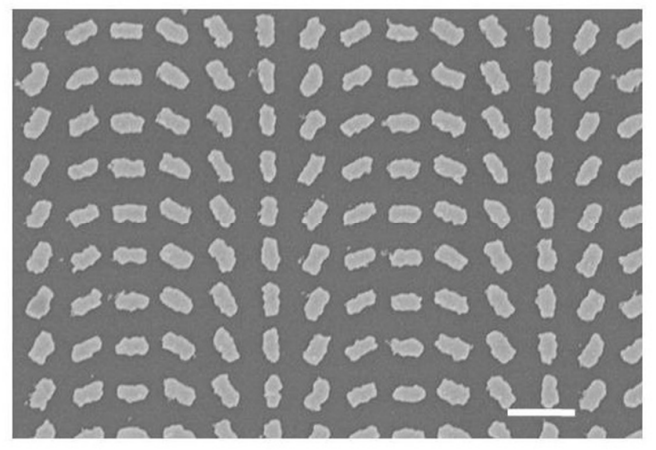


Figure S4. The SEM image of fabricated phase-gradient metasurface. The scale bar is $500 \mathrm{~nm}$.

Supplementary Section 5. SEM image of metasurface with $\alpha_{0}=\pi$
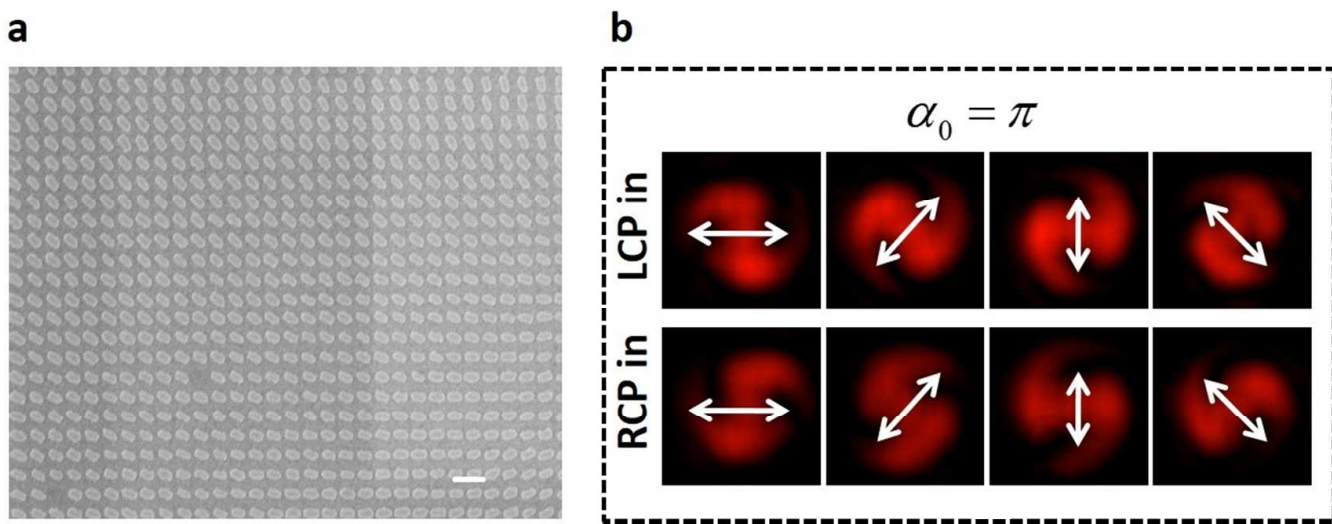

Figure S5. SEM image of metasurface with $\alpha_{0}=\pi$. Scale bar, $500 \mathrm{~nm}$.

\section{ACKNOWLEDGMENTS}

This work is supported by the Engineering and Physical Sciences Research Council of the United Kingdom (Grant Ref: EP/M003175/1).

\section{AUTHOR INFORMATION}

\section{Corresponding Author}

* Email: x.chen@hw.ac.uk

Note: F. Yue and D. Wen contribute equally to this work.

\section{REFERENCES}

(1) Zheng G, Muhlenbernd H, Kenney M, Li G, Zentgraf T, Zhang S. Metasurface holograms reaching $80 \%$ efficiency. Nature nanotechnol. 2015, 10, 308-312.

(2) Goldstein D. Polarized light. Book, 2003.

(3) Bomzon Z, Biener G, Kleiner V, Hasman E. Radially and azimuthally polarized beams generated by space-variant dielectric subwavelength gratings. Opt. Lett. 2002, 27, 285-287.

(4) Yu NF, et al. Light Propagation with Phase Discontinuities: Generalized Laws of Reflection and Refraction. Science 2011, 334, 333-337. 
